\title{
The CatLet score: a new coronary angiographic scoring tool accommodating the variable coronary anatomy for the first time
}

\author{
Ming-Xing Xu ${ }^{1}$, Ruo-Ling Teng ${ }^{1}$, Terrence D. Ruddy ${ }^{2}$, Paul Schoenhagen ${ }^{3}$, Thomas Bartel ${ }^{4}$, \\ Roberto Di Bartolomeo ${ }^{5}$, Olcay Aksoy ${ }^{6}$, Milind Desai $^{7}$, Yskert von Kodolitsch ${ }^{8}$, Javier Escaned $^{9}$, \\ Peter A. McCullough ${ }^{10}$, Anupama Vasudevan ${ }^{10}$, Cheng-Xing Shen ${ }^{11}$, Xin Zhao ${ }^{1}$, Ya-Feng Zhou ${ }^{1}$, \\ Hai-Feng Xu ${ }^{1}, \mathrm{Xu}-J i e$ Cheng ${ }^{1}$, Yong-Ming $\mathrm{He}^{1}$; written on behalf of the AME Interventional Cardiology \\ Collaborative Group
}

\begin{abstract}
${ }^{1}$ Division of Cardiology, First Affiliated Hospital of Soochow University, Suzhou 215006, China; ${ }^{2}$ Division of Cardiology, Heart Institute, University of Ottawa, Ottawa, Canada; ${ }^{3}$ Imaging Institute, Cleveland Clinic, Cleveland, OH, USA; ${ }^{4}$ Department of Cardiovascular Medicine, Heart \& Vascular Institute, Cleveland Clinic Abu Dhabi, Abu Dhabi, UAE; ${ }^{5}$ Cardio-Thoracic and Vascular Department, Division of Cardiac Surgery, S. Orsola Hospital, University of Bologna, Bologna, Italy; ${ }^{6}$ Division of Cardiology, David Geffen School of Medicine, University of California Los Angeles, Los Angeles, CA, USA; ${ }^{7}$ Department of Cardiovascular Medicine, Heart \& Vascular Institute, Cleveland Clinic, Cleveland, OH, USA; ${ }^{8}$ Department of Cardiology, University Hospital Hamburg-Eppendorf, Hamburg, Germany; ${ }^{9}$ Department of Cardiology, Hospital San Carlos, Madrid, Spain; ${ }^{10}$ Division of Cardiology, Heart \& Vascular Institute, Baylor University Medical Center, Dallas, TX, USA; ${ }^{11}$ Department of Cardiology, Shanghai Sixth People's Hospital Affiliated to Shanghai Jiao Tong University, Shanghai 200233, China

Contributions: (I) Conception and design: YM He, MX Xu; (II) Administrative support: YM He; (III) Provision of study materials or patients: YM He; (IV) Collection and assembly of data: YM He, CX Shen, X Zhao, YF Zhou, HF Xu, XJ Cheng; (V) Data analysis and interpretation: YM He, MX $\mathrm{Xu}$, RL Teng; (VI) Manuscript writing: All authors; (VII) Final approval of manuscript: All authors.

Correspondence to: Yong-Ming He, MD, PhD. The First Affiliated Hospital of Soochow University, 188, Shizi Ave., Gusu District, Suzhou 215006, China. Email: heyongming@suda.edu.cn or heyongming@catletscore.com.
\end{abstract}

Background: The SYNTAX score for decision makings or outcome predictions in coronary artery disease does not account for the variations in the coronary anatomy, which is a clear fallacy for patients with less typical anatomy than suggested by the SYNTAX score. The current study aimed to derive a new coronary angiographic scoring system accommodating the variability in the coronary anatomy.

Methods: The 17-myocardial segment model and laws of competitive blood supply and flow conservation were utilized to derive this new scoring system.

Results: We obtained 6 types of RCA dominance, 3 types of diagonal size and 3 types of left anterior descending artery (LAD) length, which together resulted in a total of 54 patterns of coronary artery circulation to account for the variability in the coronary anatomy among individuals. A Coronary Artery Tree description and Lesion EvaluaTion (CatLet) angiographic scoring system has been designed based on the above-mentioned reclassification scheme (htpp://www.catletscore.com, IE browser is required to run this calculator).

Conclusions: This new CatLet angiographic scoring system accommodated the variability in the coronary anatomy and standardized the collection of the coronary angiographic data, which could facilitate the comparison and exchange of these data between different catheter labs. Its utility for predicting the clinical outcomes and standardizing the angiographic data collection will be investigated in a series of clinical trials enrolling "all-comers" with coronary artery disease (CAD).

Keywords: Angiographic scoring; variable coronary anatomy; 17-myocardial segment model

Submitted Nov 11, 2019. Accepted for publication Nov 28, 2019.

doi: $10.21037 /$ jtd.2019.12.18

View this article at: http://dx.doi.org/10.21037/jtd.2019.12.18

(c) Journal of Thoracic Disease. All rights reserved. 


\section{Introduction}

Coronary flow-limiting lesions and their relative importances have been considered a primary determinant of survival in patients with coronary artery diseases (CAD) (1-3). Therefore, therapeutic strategy selection and outcome prediction would likely be improved by risk-scores being able to fully account for coronary anatomy variations. However, data in this regard are lacking.

In the Leaman score, the weighting factors of coronary segments are assigned according to the proportionate blood flow directed to left anterior descending artery (LAD), left circumflex (LCX) or right coronary artery (RCA), wherein coronary dominance is simply divided into right or left (4). Thus, the Leaman score is far from reflecting the variability in coronary anatomy. The SYNTAX (SYNergy between PCI with TAXUS ${ }^{\text {TM }}$ and Cardiac Surgery) score, based on this Leaman score, therefore has significant limitations to accommodate the variability in the coronary anatomy (5). A case in point is that, according to the SYNTAX score, the weighting factor of RCA is constantly 1.0 in right RCA dominant circulation, regardless of its size. However, its size and distribution vary greatly among individuals. A Coronary Artery Tree description and Lesion EvaluaTion (CatLet) angiographic scoring system has therefore been designed to reflect this variability in the coronary anatomy.

\section{Acknowledged rules and classifications}

This CatLet score has been developed based on the following rules and classifications:

The American Heart Association (AHA) classification of the coronary artery segments (6);

17-myocardial segment model (7);

Competitive coronary blood supply;

Law of flow conservation (8);

The SYNTAX score (9).

\section{Definition of the coronary tree segments}

The definition of the coronary tree segments is based on the classification proposed by the AHA (10) and further modified for this CatLet angiographic scoring system (Figure 1).

* Seg 1. RCA proximal: adopted as in the SYNTAX score;

* Seg 2. RCA mid: adopted as in the SYNTAX score;
* Seg 3. RCA distal: adopted as in the SYNTAX score;

* Seg 4. Posterior descending artery (PDA): adopted as in the SYNTAX score;

* Seg 16. Main posterolateral vessels (PLVs) from RCA: adopted as in the SYNTAX score.

- Seg 16a. First PLV of the same size from segment 16.

- Seg 16b. Second PLV of the same size from segment 16.

* Seg 16X. Major PLV from segment 16 regardless of its origin.

* Seg 16S. Minor PLV from segment 16 regardless of its origin with a preset segment difference of 0.5 compared with the major PLV.

- Seg 16\&. Only PLV from segment 16.

* Seg 16c. Obtuse marginal from segment 16, supplying a portion of inferolateral or even anterolateral wall.

- Seg 5. Left main (LM): adopted as in the SYNTAX.

* Seg 6. Proximal LAD: first third of LAD from its origin to the apex, usually ending at the first major septal branch.

* Seg 7 series. Middle LAD: second third of LAD from its origin to the apex.

* Seg 8. Distal LAD: remaining third of LAD from its origin to the apex, usually wrapping around the apex.

* Seg 9a. First diagonal (Dx) of the same size from Seg 6 or 7.

- Seg 9b. Second Dx of the same size from Seg 6 or 7.

* Seg 9X. Major Dx from Seg 6 or 7 regardless of its origin.

* Seg 9S. Minor Dx from Seg 6 or 7 regardless of its origin, with a preset segment difference of 0.5 compared with the Seg 9X.

* Seg 9\&. Only Dx usually from Seg 6 or 7.

* Seg 7\&. Mid LAD giving rise to the Seg 9\&.

* Seg 7X. Mid LAD giving rise to the Seg 9X.

* Seg 7S. Mid LAD giving rise to the Seg 9S.

* Seg 7E. Mid LAD giving rise to the Seg 9a or Seg 9b.

* Seg 7. Mid LAD giving no rise to any Dx.

* Seg 11. Proximal LCX: adopted as in the SYNTAX score.

* Seg 12a. First obtuse marginal (OM) of the same size from LCX running over the general area of the obtuse margin of the heart.

* Seg 12b. Second OM of the same size from LCX running in the same direction as Seg 12a.

- Seg 12X. Major OM from LCX running over the general area of the obtuse margin of the heart regardless of its origin.

* Seg 12S. Minor OM from LCX running in the same 
A

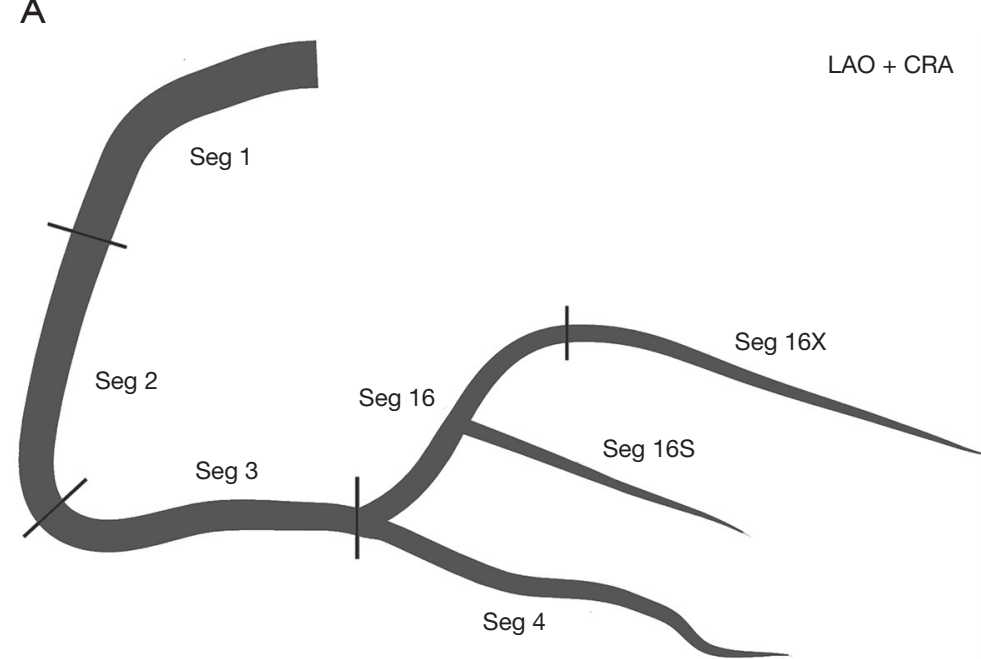

B

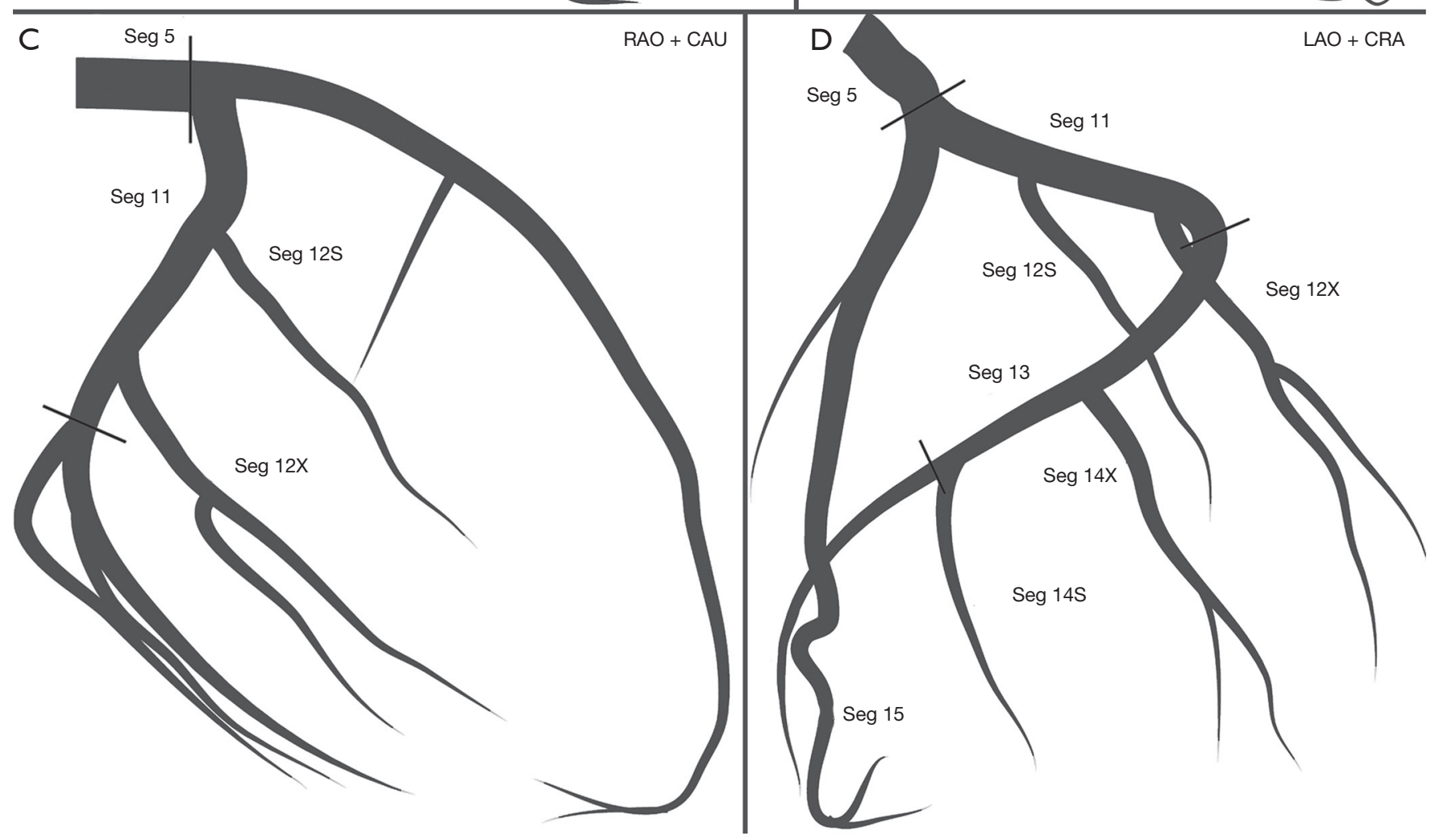

Figure 1 Definition of coronary tree segments. LAO, left anterior oblique; CRA, cranial; RAO, right anterior oblique; CAU, caudal.

direction as the Seg $12 \mathrm{X}$ regardless of its origin, with a preset segment difference 0.5 compared with the Seg $12 X$.

* Seg 12\&. Only OM from LCX running over the general area of the obtuse margin of the heart.

* Seg 13. Distal LCX: adopted as in the SYNTAX score.
* Seg 14a: First PLV of the same size from Seg 13.

* Seg 14b: Second PLV of the same size from Seg 13.

* Seg 14X: Major PLV from Seg 13 regardless of its origin.

* Seg 14S: Minor PLV from Seg 13 regardless of its origin, with a preset segment difference of 0.5 compared with Seg 14 S. 
* Seg 14\&: Only PLV from Seg 13.

* Seg 15: PDA from left coronary artery (LCA) adopted as in the SYNTAX score.

\section{7-myocardial segment model and proposal of a benchmark type of coronary circulation and its weighting factor assignment}

The 17-myocardial segment model is based on the approximately even division of the left ventricle (LV) (7), each segment can thus be understood either as 1 mass unit or 1 actual flow unit given the actual myocardial blood is proportional to muscle mass (11). The importance of a blood vessel (weighting factor) depends on how many segments the blood vessel supplies rather than on the nomenclature of the blood vessel.

Although there is variability in the coronary artery blood supply to the myocardial segments, it is generally considered appropriate to assign individual segments to specific coronary artery territories, which is defined as the benchmark type in this study (12). The assignment of the 17 -myocardial segments to one of the 3 major coronary arteries is recommended by AHA Scientific Statement as follows: LAD supplies 7 segments: 1, 2, 7, 8, 13, 14, and 17; LCX supplies 5 segments: 5, 6, 11, 12, and 16; and RCA supplies 5 segments: 3, 4, 9, 10, and 15. Correspondingly, weighting factors are 7.0 for LAD, 5.0 for LCX, and 5.0 for RCA in this benchmark type. Specifically, LAD divides into LADex (excluding diagonals) and diagonals, supplying 4 segments and 3 segments, respectively; RCA divides into PDA and PLVs, supplying 2 segments and 3 segments, respectively. Correspondingly, weighting factors are 3.0 for diagonals, 2.0 for PDA, and 3.0 for PLVs in this benchmark type.

\section{Competitive coronary blood supply accounts for the variability in the coronary artery tree among individuals}

RCA and LCA provide competitive patterns of blood supply for the entire LV. Specifically, LAD competes with PDA; diagonals compete with OMs off LCX; and PLVs off RCA competes with PLVs off LCX in supplying the LV. A large RCA is associated with a small LCA; large diagonals are associated with small obtuse marginal arteries; large PLVs off RCA are associated with small PLVs off LCX; a long LAD is associated with a short PDA, and vice versa. We propose a reclassification scheme of the coronary artery circulation pattern in order to reflect this variability.

\section{Reclassification of RCA dominance}

The size of RCA is defined depending on the distribution of the LCX according to the rule of competitive blood supply. The stem LCX (not its branches) usually runs in the left atrio-ventricular sulcus (LAVS). Therefore, the stem LCX's course on the cineangiograms can be utilized to indicate the LAVS. The crux cordis approximately falls upon the perpendicular line via the aorta ostium of the LCA (aorta wall). The crux cordis is the intersection point of the perpendicular line with the LAVS. In the left anterior oblique (LAO) $40^{\circ}$ cranial $20^{\circ}$ view, LAVS runs from the upper right to the lower left, starting at the junction of the first quarter of the leftmost margin of the left heart with an approximately $90^{\circ}$ angle referring to the left heart margin and terminating at the crux cordis (Figure 2, Figure 2M).

* PDA zero (left dominance): RCA gives no rise to any branches supplying the LV. The entire LV is supplied by LCA (Figure $2 A$ ).

- PDA only: the stem LCX gives rise to a significant branch from the last third of the LAVS immediately next to the crux cordis to supply the entire inferior wall in the LAO $40^{\circ}$ /cranial $20^{\circ}$ view. RCA provides only blood flow to the inferior septum (Figure $2 B$ ).

* Small RCA: the stem LCX gives rise to a significant branch from the second third of the LAVS to supply a portion of the inferior wall in the LAO $40 \%$ cranial $20^{\circ}$ view. RCA provides blood flow to the inferior septum and the remaining portion of the inferior wall (Figure 2C).

* Average RCA: the stem LCX gives rise to a significant branch from the first third of the LAVS to supply the inferolateral wall in the LAO $40^{\circ}$ cranial $20^{\circ}$ view. RCA provides blood flow to the inferior septum and the entire inferior wall (Figure 2D).

* Large RCA: the stem LCX only gives rise to branches to supply the anterolateral wall in the LAO $40^{\circ}$ /cranial $20^{\circ}$ view. RCA provides blood flow to the inferior septum, the inferior wall and the inferolateral wall (Figure 2E).

* Super RCA: the stem LCX gives rise to relatively small branches to supply a portion of the anterolateral wall in the $\mathrm{LAO} 40^{\circ} \%$ cranial $20^{\circ}$ view. RCA provides blood flow to the inferior septum, the inferior wall, the inferolateral wall and the remaining portion of the anterolateral wall (Figure $2 F$ ). 

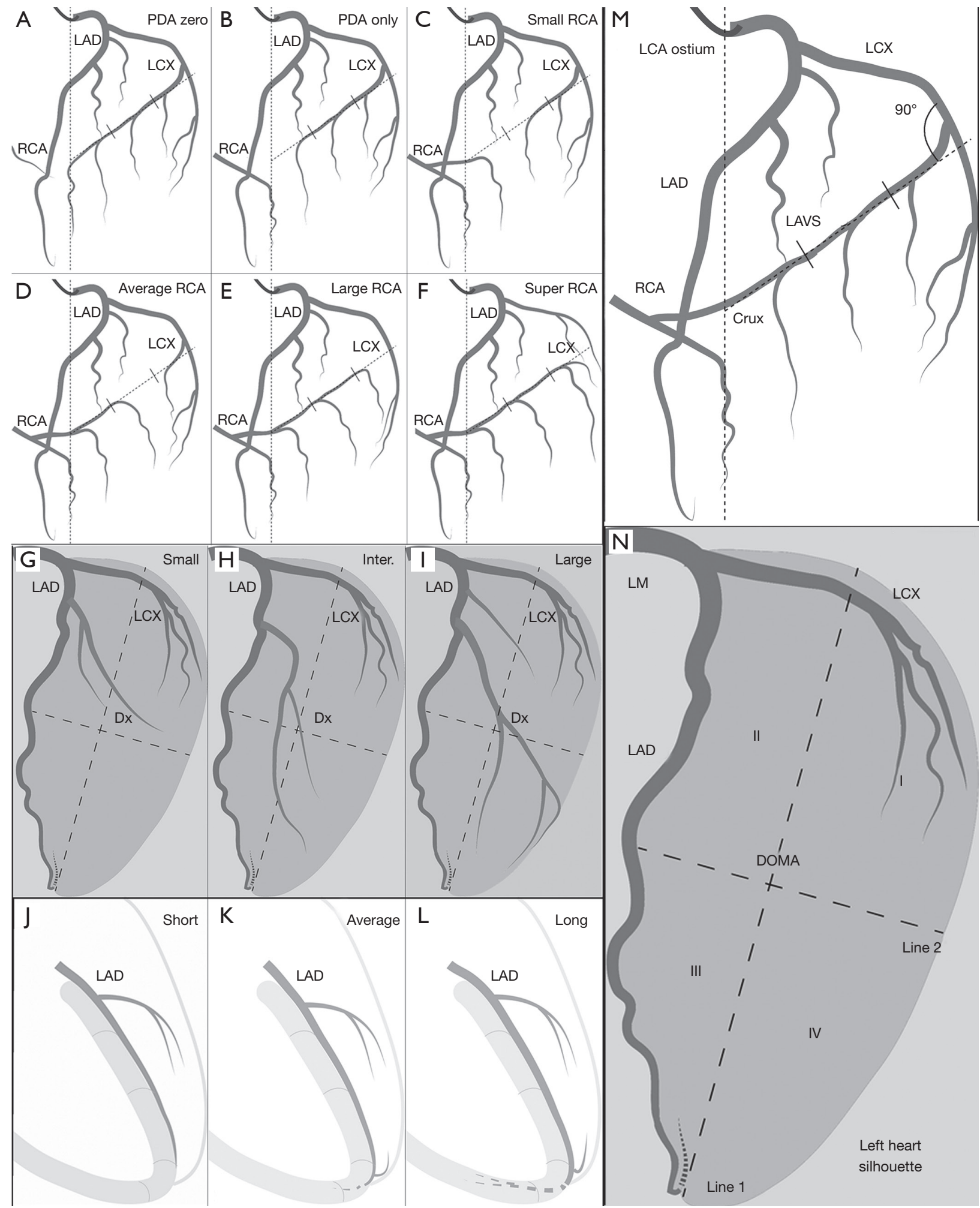

Figure 2 Reclassification scheme accommodating the variable coronary anatomy. PDA, posterior descending artery; RCA, right coronary artery; LCA, left coronary artery; LCX, left circumflex; RCA, right coronary artery; LAVS, left atrio-ventricular sulcus; LM, left main; $\mathrm{LAD}$, left anterior descending artery; DOMA, diagonal-obtuse marginal area. 


\section{Reclassification of Dx size}

The diagonal-obtuse marginal area (DOMA) of the LV is competitively perfused by both Dx off LAD and OM off LCX. In the LAO $40^{\circ}$ cranial $20^{\circ}$ view, the DOMA takes on the approximate shape of a spindle delineated by discernable anatomic markers on coronary angiograms. Before giving rise to obtuse marginal arteries, initial stem LCX courses usually transversely in the LAVS and delineates the upper border of the DOMA. LAD almost constantly courses in the anterior interventricular sulcus and delineates the right boundary of the DOMA. The DOMA's left and inferior margins are recognizable because of sufficient contrast between the heart margin and its surrounding tissues on cineangiograms. An imaginary line 1 starting from the apex divides the DOMA into 2 similar portions. Another imaginary line 2 perpendicular to the line 1 at its midpoint also divides the DOMA into 2 portions. Thus, we get 4 quadrants counterclockwise over the DOMA: I, II, III, and IV as shown in Figure 2N.

* Small Dx: Dx distributing over $\leq 2$ quadrants with approximately 1.5 or less segments (Figure $2 G$ ).

* Inter. Dx: Dx distributing over 3-4 quadrants with approximately $1.5-3$ segments (Figure $2 H$ ).

* Large Dx: Dx distributing over $>4$ quadrants in excess of 3 segments (Figure 2I).

\section{Reclassification of LAD length}

* Short LAD: LAD terminates before the apex and supplies the entire anterior septum with approximately 3 segments (Figure 27);

* Average LAD: LAD reaches the apex and supplies the whole anterior septum and the apex with approximately 4 segments with a terminal shape of inverted $Y^{\prime}$ (Figure 2K);

* Long LAD: LAD turns around the apex notch and runs further in the posterior interventricular sulcus, supplying the whole anterior septum, apex and a portion of posterior septum with approximately 5 segments (Figure 2L).

Six types of RCA dominance, 3 types of Dx size, and 3 types of LAD length together result in a total of 54 patterns of coronary artery circulation, which are used to account for the variability in coronary anatomy among individuals.

According to the law of flow conservation (8), a parent vessel equals the sum of its daughter vessels in terms of blood flow. Weighting factor assignment strictly follows this law. Based on the standard 17 segment model of the $\mathrm{LV}$, the weighting factors are derived from the abovedescribed anatomic details of RCA, LAD, LCX or their branches (variations in coronary anatomy). The benchmark pattern of coronary circulation is used as reference and weighting factors with discrete values are added to account for the variations in the circulation pattern. Use of preset, discrete incremental values is a compromise between the convenience of clinical use and the variability in coronary artery circulation. A preset incremental value of 1 or 1.5 was used to describe differences in LAD length, in diagonal size or in RCA supply territory. This approach, in combination with the rule of competitive blood supply and the law of flow conservation (8), allows to derive the weighting factors as shown in Figure 3 for all the coronary artery segments in the 54 patterns of coronary artery circulation. Based on these weighting factors, we developed a new CatLet angiographic scoring system accounting for the variability in the coronary anatomy.

\section{Lesion scoring and the need for adapting modifications}

The lesion score was a product of the weighting factor $(\mathrm{W})$ of a blood vessel and its degree of stenosis (9). Separate lesion scores were then added to derive the total score. In this CatLet scoring system, only lesions with $\geq 50 \%$ diameter stenosis on vessels $>1.5 \mathrm{~mm}$ in diameter were scored while all the non-stenosis adverse characteristics pertinent to the lesions were no longer scored, but only qualitatively recorded.

Within one segment, however, direct scoring will overestimate the severity of coronary artery disease in the presence of side branches preceding the main branch (MB) lesion regardless of their normal or diseased status. We will explain the need for adapting modifications with the scenario 1 as listed below (Figure 4). In this scenario, suppose the weighting factor is 7.0 for the segment and 3.0 for the side branch (SB). The MB of the segment divides into proximal or distal MB referring to the SB. According to the law of flow conservation, the weighting factor for the distal MB is 4.0. Within one segment, an occlusive lesion for this segment distal to the $\mathrm{SB}$ is scored as $7.0 \times 5=35.0$ according to the CatLet scoring algorithm. Actually, the distal MB lesion's weighting factor is only 4.0 because of the presence of the SB preceding the occlusion lesion. Hence, the CatLet scoring algorithm overestimates the severity of 


\begin{tabular}{|c|c|c|c|c|c|c|c|c|c|c|c|c|c|c|c|c|c|c|c|c|c|c|c|}
\hline & 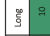 & & & & & 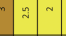 & $\frac{2}{2} / \mathscr{Z}$ & & 경 & & & $\frac{\vec{z}}{2} \frac{\vec{z}}{\frac{1}{2}}$ & & & & & & & & & & & \\
\hline 萨 & $\frac{1}{3}$ & & & & $\approx$ & . 17 & ‡) & & za & 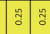 & 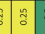 & $\frac{\vec{t}}{2} \frac{\vec{z}}{2}$ & 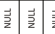 & $\frac{\vec{y}}{\underline{\underline{z}}}$ & & $\frac{\vec{z}}{\underline{z}}$ & & & & 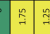 & & & \\
\hline & \begin{tabular}{|l|l|}
5 \\
s.
\end{tabular} & & & & 7 & $\therefore 2$ & $\beth \beth$ & & \begin{tabular}{|l|l|} 
\\
\end{tabular} & z: & 8 & \begin{tabular}{l|l}
$\frac{\hat{z}}{2}$ & $\underline{\underline{z}}$ \\
\end{tabular} & 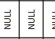 & 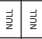 & & & & & & 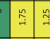 & $\because$ & & \\
\hline & ฐ̊ & & & & $\because$ & $5 \cong$ & $\because=$ & & 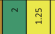 & $\frac{\mathrm{m}}{\mathrm{g}}$ & & \begin{tabular}{l|l}
$\vec{z}$ & $\underline{\underline{z}}$ \\
\end{tabular} & $\frac{\vec{a}}{\vec{z}} \frac{\vec{t}}{\bar{z}}$ & $\frac{\vec{b}}{\frac{\vec{z}}{2}}$ & & $\overrightarrow{\underline{\underline{z}}}$ & & & & $\frac{2}{2}$ & & & \\
\hline & $\frac{1}{2}$ & & & & 2 & $\because \exists$ & 77 & & & $\frac{1}{6}$ & 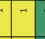 & \begin{tabular}{|l|l|l}
$\frac{\vec{z}}{2}$ & $\underline{\underline{z}}$ \\
\end{tabular} & $\frac{\vec{z}}{\bar{z}} \frac{\bar{t}}{\bar{z}}$ & 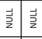 & $\frac{1}{z}$ & $\frac{1}{2}$ & & & & $\stackrel{2}{\prime}$ & & & \\
\hline & \begin{tabular}{|l|l|}
5 \\
5
\end{tabular} & & & & & $\because 7$ & $\eta \cong$ & & $\nexists$ & $\begin{array}{ll}\frac{m}{6} \\
\end{array}$ & & 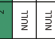 & $\frac{\vec{z}}{\bar{z}} \overline{\frac{1}{z}}$ & \begin{tabular}{l|l}
$\vec{z} \frac{\vec{z}}{2}$ & $\frac{\vec{z}}{2}$ \\
\end{tabular} & $\frac{\vec{z}}{2}$ & $\frac{\vec{z}}{\underline{z}}$ & & & & $\cong$ & $=$ & & \\
\hline & פّ & & & & & & $5: 5$ & & & $\Rightarrow$ & $\stackrel{2}{\square}$ & $\overrightarrow{\frac{\vec{z}}{2}}$ & $\overrightarrow{\vec{z}} \bar{z} \frac{\vec{z}}{\vec{z}}$ & \begin{tabular}{l|l}
$\overrightarrow{\underline{z}}$ & $\overrightarrow{\underline{z}}$ \\
\end{tabular} & $\frac{\vec{z}}{\vec{z}}$ & & & & & $\cong$ & & & \\
\hline 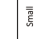 & \begin{tabular}{|l|l|}
$\frac{1}{2}$ \\
\end{tabular} & & & & $\stackrel{n}{n}$ & 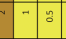 & $5: 5$ & & $\sim$ & $\Rightarrow g$ & 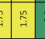 & 童言 & $\begin{array}{lll}\vec{z} & \frac{1}{z} \\
\end{array}$ & \begin{tabular}{l|l}
$\frac{1}{2}$ & $\frac{1}{2}$ \\
\end{tabular} & $\frac{\vec{z}}{2}$ & $\frac{3}{2}$ & & & & $\cong$ & & & \\
\hline & 喜 & 8 & & & 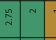 & $-a$ & $\div: 5$ & & $\sim$ & $\Rightarrow \cong$ & $\because \frac{\pi}{\square}$ & \begin{tabular}{l|l}
$\frac{3}{2}$ & $\frac{3}{2}$ \\
\end{tabular} & $\frac{\overrightarrow{3}}{2} \frac{\vec{t}}{\frac{3}{2}}$ & 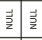 & $\frac{\vec{z}}{2}$ & $\overrightarrow{\underline{z}}$ & & & & $\cong$ & $\because$ & & \\
\hline & פू & $a:$ & & & $:$ & \begin{tabular}{|l|l|}
2 & \\
\end{tabular} & $\beth \beth$ & & $\nexists$ & \begin{tabular}{|c|c|}
8 & -1
\end{tabular} & 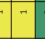 & \begin{tabular}{l|l}
$\vec{t}$ \\
$\frac{\vec{z}}{2}$
\end{tabular} & 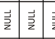 & \begin{tabular}{l|l}
$\vec{z}$ & $\vec{z}$ \\
\end{tabular} & $\frac{\vec{z}}{2}$ & $\vec{z}$ & & & & 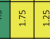 & & & \\
\hline 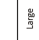 & \begin{tabular}{|l|l|}
$\frac{1}{2}$ \\
\end{tabular} & 2 & & & 2 & \begin{tabular}{|l|l|} 
& \\
\end{tabular} & $\nexists \nexists$ & & & $\begin{array}{ll}n \\
\end{array}$ & -7 & \begin{tabular}{l|l}
$\vec{z}$ \\
$\underline{z}$
\end{tabular} & $\frac{\vec{z}}{\vec{z}} \overline{\underline{z}}$ & \begin{tabular}{l|l}
$\vec{z}$ \\
$\underline{2}$
\end{tabular} & & $\underline{\underline{3}}$ & & 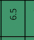 & & $\cong$ & & & \\
\hline & \begin{tabular}{|l|l|} 
喜 \\
\end{tabular} & $\cong$ & & & $\mathrm{z}$ & \begin{tabular}{|l|l|} 
& \\
\end{tabular} & $\approx \approx$ & & $\nexists$ & \begin{tabular}{|l|l}
5 & -1
\end{tabular} & 17 & 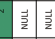 & 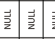 & 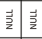 & $\frac{3}{\frac{3}{2}}$ & $\frac{3}{2}$ & & & & $\cong$ & & & \\
\hline & $\underline{\xi}$ & & & 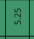 & 2 & $\because \cong$ & $2=$ & & $\sim$ & 29 & 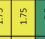 & $\frac{\vec{z}}{\vec{z}} \frac{\vec{z}}{\vec{z}}$ & $\frac{\vec{z}}{\vec{z}} \overrightarrow{\underline{z}}$ & \begin{tabular}{l|l}
$\overrightarrow{\underline{z}}$ \\
$\underline{\underline{z}}$
\end{tabular} & & $\overrightarrow{\underline{z}}$ & & 2 & & $\cong$ & & & \\
\hline 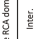 & \begin{tabular}{|l|l|}
1 \\
\end{tabular} & & & $\stackrel{7}{7}$ & 7 & $\because 9$ & 72 & & $\sim$ & 72 & 9 & \begin{tabular}{l|l}
$\frac{\vec{z}}{2}$ \\
\end{tabular} & 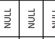 & 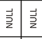 & $\frac{1}{2}$ & $\frac{3}{2}$ & & $\because$ & & $\cong$ & $=$ & & \\
\hline & \begin{tabular}{|l|l|} 
喜 \\
\end{tabular} & & & & 2 & $\Xi \because$ & $7 \%$ & & & $\cong \cong$ & 5 & \begin{tabular}{l|l}
$\frac{\vec{t}}{2}$ & $\frac{\vec{t}}{2}$ \\
\end{tabular} & 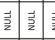 & \begin{tabular}{l|l}
$\vec{z} \frac{\vec{z}}{2}$ \\
\end{tabular} & $\frac{\vec{z}}{\frac{3}{2}}$ & $\frac{\vec{z}}{\vec{z}}$ & & & & $\cong$ & & & \\
\hline & 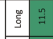 & $5:$ & & & 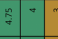 & 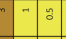 & $5: 5$ & & $\begin{array}{ll}n \\
2\end{array}$ & 22 & $\approx$ & 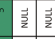 & $\frac{\vec{a}}{2} \frac{\vec{b}}{2}$ & 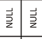 & $\frac{1}{2}$ & $\frac{\vec{z}}{\overrightarrow{2}}$ & & 4 & & \begin{tabular}{|l|l}
$\cong$ & $\varrho$
\end{tabular} & & & \\
\hline$\overline{\bar{n}}$ & \begin{tabular}{|l|l|}
3 \\
\end{tabular} & 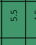 & $\frac{7}{8}$ & 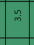 & $\begin{array}{ll}\mathrm{n} \\
\mathrm{n}\end{array}$ & $-:$ & $\div 5$ & & $\frac{n}{2}$ & 22 & 2 & \begin{tabular}{|l|l|l|}
$\vec{z}$ & $\underline{\underline{z}}$ \\
\end{tabular} & $\begin{array}{l}\overrightarrow{\underline{z}} \bar{z} \\
\bar{z}\end{array}$ & \begin{tabular}{l|l|l}
$\vec{z}$ & $\vec{z}$ \\
\end{tabular} & $\frac{\vec{z}}{\bar{z}}$ & $\vec{z}$ & & 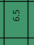 & & 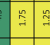 & $\because$ & & \\
\hline & \begin{tabular}{|l|}
\multicolumn{5}{|c|}{} \\
\end{tabular} & 7 & & $\Rightarrow$ & 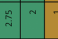 & $7:$ & $\frac{1}{2} 5 \%$ & & $\begin{array}{ll}n \\
N\end{array}$ & 22 & 2 & \begin{tabular}{|l|l}
$\frac{\vec{z}}{2}$ & $\frac{\vec{z}}{z}$ \\
\end{tabular} & $\frac{\vec{z}}{\bar{z}} \overline{\frac{1}{z}}$ & \begin{tabular}{l|l}
$\overrightarrow{\frac{1}{2}}$ & $\frac{\vec{t}}{2}$ \\
\end{tabular} & $\frac{\vec{z}}{2}$ & $\underline{\underline{z}}$ & & 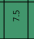 & & $\stackrel{2}{\beth}$ & & & \\
\hline & \begin{tabular}{|l|}
\multirow{5}{5}{} \\
\end{tabular} & $:$ & & & 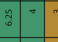 & \begin{tabular}{|l|l|} 
& \\
\end{tabular} & $\nexists \nexists$ & & & $\Rightarrow 2$ & 2 & 考妾 & 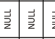 & 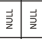 & $\frac{1}{2}$ & $\underline{3}$ & & & & $\cong \cong$ & & & \\
\hline 罗 & $\frac{1}{2}$ & ${ }_{\infty}^{2}$ & & & $\pi$ & \begin{tabular}{|c|c|} 
& $\sim$ \\
\end{tabular} & $\approx \approx$ & & $\sim$ & $\Rightarrow \cong$ & $\frac{2}{\pi}$ & \begin{tabular}{|l|l}
$\vec{z}$ \\
$\underline{\underline{z}}$
\end{tabular} & $\frac{\vec{z}}{\bar{z}} \overline{\frac{1}{2}}$ & \begin{tabular}{l|l}
$\overrightarrow{\underline{z}} \mid \vec{z}$ \\
\multirow{2}{*}{}
\end{tabular} & $\frac{1}{2}$ & & & 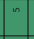 & & \begin{tabular}{l|l}
$\infty$ & $\infty$ \\
$\sim$ &
\end{tabular} & & & \\
\hline & \begin{tabular}{|l|l|} 
\\
\end{tabular} & 2 & & & $\exists$ & $2 \pi$ & I & & & $\Rightarrow 8$ & 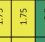 & 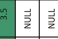 & 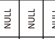 & \begin{tabular}{l|l}
$\overrightarrow{\vec{z}}$ & $\overrightarrow{\underline{z}}$ \\
\end{tabular} & $\frac{3}{z}$ & & & & & $\Xi \Xi$ & & & \\
\hline & 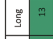 & ${ }^{\infty}$ & & 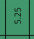 & " & $\because \cong$ & 77 & & 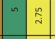 & 27 & 27 & 旁妾 & $\frac{\vec{z}}{2} \overline{\frac{1}{2}}$ & \begin{tabular}{l|l}
$\vec{z} \underline{z}$ & $\underline{\underline{z}}$
\end{tabular} & $\frac{1}{2}$ & $\frac{1}{z}$ & & + & & $\cong$ & $\because$ & & \\
\hline 竞 & \begin{tabular}{|l|l|}
$\frac{1}{2}$ \\
\end{tabular} & & $\circ$ & 3 & ? & \begin{tabular}{l|l|l|l|}
$n$ & $\cong$
\end{tabular} & $7 \%$ & & \begin{tabular}{|l|l|} 
\\
\end{tabular} & 22 & 9 & \begin{tabular}{|l|l}
$\vec{z}$ \\
$\underline{\underline{z}}$
\end{tabular} & $\frac{\vec{z}}{\overline{2}} \frac{\overrightarrow{\mid}}{\bar{z}}$ & \begin{tabular}{l|l}
$\overrightarrow{\underline{z}}$ & $\overrightarrow{\underline{z}}$ \\
\end{tabular} & $\frac{\vec{z}}{2}$ & $\underline{\underline{z}}$ & & 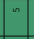 & & 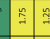 & & & \\
\hline & 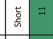 & & & $\%$ & $m$ & 27 & 77 & & $\stackrel{n}{I}$ & 2 . & จ & $\overrightarrow{\underline{z}} \frac{\vec{z}}{\vec{z}}$ & $\overrightarrow{\underline{z}} \frac{\vec{z}}{\vec{z}}$ & \begin{tabular}{l|l}
$\overrightarrow{\underline{z}}$ & $\overrightarrow{\underline{z}}$ \\
\end{tabular} & $\frac{\vec{z}}{\vec{z}}$ & $\frac{\vec{z}}{\vec{z}}$ & & 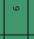 & & 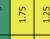 & & & \\
\hline & פू & $y=$ & & 7 & \begin{tabular}{|l|l|l|}
7 \\
\end{tabular} & 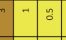 & $5:$ & & 2 & $m \nexists$ & $2 \approx$ & \begin{tabular}{l|l}
$\vec{z}$ \\
\end{tabular} & $\frac{\vec{z}}{\bar{z}} \overline{\underline{z}} \frac{3}{3}$ & 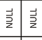 & 青 & 产 & & 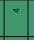 & & 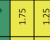 & $\simeq$ & & \\
\hline$\overline{\bar{z}}$ & \begin{tabular}{|l|l|}
$\frac{3}{2}$ \\
\end{tabular} & $:=$ & 7 & $\begin{array}{ll} \\
\end{array}$ & 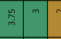 & 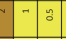 & 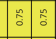 & & 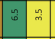 & $\because \cong$ & $\approx \approx$ & $\frac{\vec{t}}{2} \frac{\vec{a}}{2}$ & 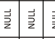 & 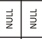 & & $\overrightarrow{\underline{z}}$ & & & & 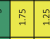 & & & \\
\hline & \begin{tabular}{|l|l} 
\\
\\
\end{tabular} & 7 & & I & $\stackrel{D}{D}$ & $\Rightarrow:$ & $5: 5$ & & $\%$ & $m$ & $2 \approx$ & 旁 & $\frac{\vec{z}}{\vec{z}} \overline{\frac{a}{z}}$ & 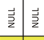 & & $\overrightarrow{\vec{z}}$ & & ${ }^{\circ}$ & & 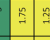 & 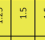 & & \\
\hline & 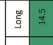 & ${ }^{2}$ & & & 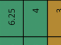 & \begin{tabular}{|l|l|}
2 \\
\end{tabular} & $\nexists 7$ & a & & $\Rightarrow \cong$ & 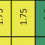 & & $-\because:$ & 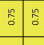 & & $\frac{\vec{z}}{\vec{z}}$ & & 2 & & & 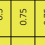 & & \\
\hline 总 & $\frac{1}{2}$ & 2 & & & 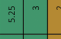 & $\approx$ & $\Xi 2$ & & & $\Rightarrow \cong$ & $\therefore$ & & 2 & \begin{tabular}{|l|l}
5 \\
\end{tabular} & & $\underline{\underline{z}}$ & & $n$ & & & $8 \%$ & & \\
\hline & \begin{tabular}{|l|l|}
$\frac{5}{\bar{n}}$ \\
\end{tabular} & 2 & & & 7 & \begin{tabular}{|l|l|} 
& \\
\end{tabular} & $\nexists \beth$ & & $\sim$ & $\Rightarrow g$ & 9 & & $\because$ & \begin{tabular}{|l|l}
5 \\
0
\end{tabular} & & $\overrightarrow{\underline{\vec{t}}}$ & & 7 & & $-"$ & 8 & & \\
\hline & 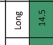 & & $\simeq$ & \begin{tabular}{|c|c} 
\\
0 \\
0
\end{tabular} & : & \begin{tabular}{|l|l|l|}
$\because$ & \\
\end{tabular} & 77 & & $\frac{n}{n}$ & $\approx \approx$ & 2 & & $\therefore$ & 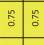 & & $\underline{\underline{z}}$ & & 2 & & & 角 & 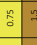 & \\
\hline ti & 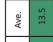 & & $\circ$ & 7 & 7 & $\because \because$ & 77 & & & $2 \approx$ & 27 & & 2 & \begin{tabular}{|l|l}
5 \\
5
\end{tabular} & & $\mid \frac{\vec{z}}{\vec{z}}$ & & 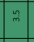 & & & $8 \%$ & $\frac{1}{8}$ & \\
\hline & \begin{tabular}{|l|l|}
\multicolumn{2}{|l|}{} \\
\end{tabular} & & n & 2 & $\therefore$ & \begin{tabular}{|l|l|}
5 & 2 \\
\end{tabular} & 72 & 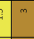 & \begin{tabular}{|l|l|}
2 \\
\end{tabular} & $2 y$ & 92 & & 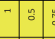 & \begin{tabular}{|l|l}
5 \\
\end{tabular} & & $\frac{\vec{z}}{2}$ & & $\eta$ & & & $3:$ & 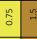 & \\
\hline & 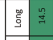 & 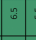 & & & $\begin{array}{ll} \\
7\end{array}$ & $7:$ & $5:$ & & $\stackrel{m}{*}$ & " & $\approx$ & & 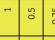 & \begin{tabular}{|l|l}
5 \\
$\vdots$
\end{tabular} & & $\underline{\underline{z}}$ & & & & & $\begin{array}{lll} \\
\end{array}$ & 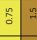 & \\
\hline 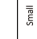 & $\frac{1}{2}$ & 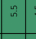 & 7 & " & $\begin{array}{ll}2 \\
0 \\
0\end{array}$ & 2 & \begin{tabular}{|l|l|l} 
& 5 \\
0 & 5 \\
\end{tabular} & & $m$ & $m$ & 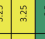 & & 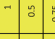 & \begin{tabular}{|l|l}
5 & $n$ \\
0
\end{tabular} & & $\overrightarrow{\underline{z}}$ & & $\%$ & & & $8 \%$ & $\frac{2}{\circ}$ & 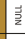 \\
\hline & \begin{tabular}{|l|l|} 
憘 \\
\end{tabular} & 7 & & & $\begin{array}{ll}2 \\
2\end{array}$ & : & 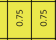 & & 9 & $m$ & 2 & & : & \begin{tabular}{|l|l}
\multirow{2}{*}{} & g \\
\end{tabular} & & $\frac{\vec{t}}{\vec{z}}$ & & $y$ & & $7=$ & $3 \%$ & 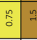 & 表 \\
\hline & 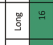 & 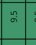 & & & \begin{tabular}{|l|l|}
\multirow{3}{*}{} \\
\end{tabular} & \begin{tabular}{|l|l|} 
& $\sim$ \\
\end{tabular} & $\approx \approx$ & & & $\Rightarrow \cong$ & 5 & & $\approx=$ & $\because \approx$ & & $\underline{\underline{z}}$ & & & & $\frac{\vec{z}}{2}$ & $\overrightarrow{3}$ & & 考 \\
\hline 岁 & $\frac{1}{2}$ & $\approx$ & & & \begin{tabular}{|l|}
$y$ \\
\end{tabular} & 2 & $\nexists \beth$ & 8 & & $\cong \cong$ & 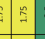 & & $\Xi \nexists=$ & 77 & & $\overrightarrow{\underline{z}}$ & & & & 考宣 & $\vec{z}$ & 言言 & $\frac{\vec{z}}{\underline{z}}$ \\
\hline & \begin{tabular}{|l|l|}
5 \\
\end{tabular} & $\Rightarrow$ & & & 2 & $2{ }^{2}$ & $\approx \beth$ & & $\sim$ & $\Rightarrow$ & 5 & & $\stackrel{2}{\because}=$ & 77 & & 昱 & & & & 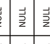 & $\frac{1}{2}$ & 言言 & 考 \\
\hline & \begin{tabular}{|l|l|}
$\underline{5}$ \\
\end{tabular} & & & \begin{tabular}{ll}
\multirow{2}{*}{} \\
s.
\end{tabular} & $\begin{array}{ll}2 \\
\text { in }\end{array}$ & 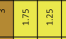 & $\Rightarrow \approx$ & & $\frac{n}{i}$ & 22 & $2 \approx$ & & $\approx$ & $\Rightarrow=$ & & $\underline{\underline{z}}$ & & & & $\frac{|l|}{2}$ & $\frac{\vec{t}}{2}$ & & 言 \\
\hline & $\frac{1}{2}$ & 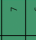 & $\circ$ & 7 & 7 & 77 & 77 & & $\frac{n}{2}$ & 27 & 2 & & $\exists=$ & $\begin{array}{lll}77 \\
\end{array}$ & & $\overrightarrow{\underline{z}}$ & & & & $\frac{1}{2}$ & $\vec{z}$ & 言言 & 声 \\
\hline & \begin{tabular}{|l|l|}
5 \\
\end{tabular} & & " & 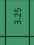 & 9 & 9 & 77 & & $\frac{n}{2}$ & $2 y$ & 27 & & $\stackrel{7}{g}$ & $\begin{array}{lll}7 & 7 \\
\end{array}$ & & 声 & & " & & $\frac{1}{2}$ & $\frac{\vec{z}}{2}$ & & 吉 \\
\hline & 泡 & $:$ & & 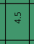 & $\frac{10}{3}$ & $-z$ & $\frac{2}{5} \%$ & & 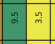 & $m$ & $\approx$ & & $\cong$ & $\because \cong$ & & \begin{tabular}{|l|}
$\underline{\underline{z}}$ \\
\end{tabular} & & 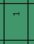 & & $\frac{1}{2}$ & $\frac{\vec{t}}{2}$ & 言言 & 景 \\
\hline 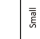 & $\frac{1}{2}$ & . & & 2 & \begin{tabular}{|l|l} 
\\
0 \\
$m$
\end{tabular} & "5 & 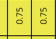 & & 2 & $m$ & 2 & & $\Xi \nexists=$ & 77 & & $\frac{\vec{t}}{\vec{z}}$ & & | & & $\frac{1}{2}$ & $\frac{\vec{z}}{\vec{z}}$ & 言竞 & $\frac{\vec{z}}{2}$ \\
\hline & \begin{tabular}{|l|l|}
$\underline{5}$ \\
\end{tabular} & ${ }_{7}^{7}$ & & $n$ & \begin{tabular}{|l|l|} 
\\
\end{tabular} & -5 & \begin{tabular}{l|l}
0 & $\circ$ \\
\end{tabular} & & $\Rightarrow$ & $m$ & 2 & $\stackrel{n}{3}$ & 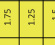 & 77 & & $\overrightarrow{\underline{z}}$ & 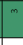 & $m$ & 言 & $\frac{1}{2}$ & $\frac{\vec{z}}{2}$ & $\frac{\overline{\underline{z}}}{\overline{2}}$ & 表 \\
\hline & $\frac{\mathscr{g}}{\underline{g}}$ & $a$ & & & \begin{tabular}{|l|l|}
5 \\
\end{tabular} & \begin{tabular}{|l|l|}
2 \\
\end{tabular} & $\nexists \approx$ & & & $\Rightarrow \cong$ & 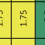 & & $\Xi \nexists=$ & 77 & & & & $\overrightarrow{\underline{z}}$ & $\underline{\underline{\underline{z}}} \overline{\underline{z}}$ & $\frac{\vec{z}}{\bar{z}}$ & $\frac{\vec{b}}{\vec{z}}$ & 言言 & 景 \\
\hline 兽 & $\frac{3}{2}$ & 2 & & & 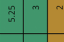 & $2 \sim$ & $\cong \beth$ & & $\sim$ & $\cong \stackrel{n}{ }$ & 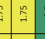 & & $\cong \cong$ & $\Rightarrow 7$ & & $\underline{\underline{\underline{z}}}$ & & $\frac{\vec{z}}{\bar{z}}$ & 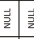 & $\frac{1}{2} \frac{\vec{z}}{2}$ & $\frac{\vec{z}}{2}$ & $\frac{\vec{z}}{\frac{\partial}{2}} \frac{\bar{z}}{\bar{z}}$ & $\frac{\vec{z}}{2}$ \\
\hline & \begin{tabular}{|l|l} 
\\
\end{tabular} & 2 & & & 7 & \begin{tabular}{|l|l|} 
& \\
\end{tabular} & ป ปิ & & & $\Rightarrow g$ & $\frac{2}{7}$ & & ‡. & \begin{tabular}{|l|l}
77 & 7 \\
\end{tabular} & & 列 & & $\underline{\underline{z}}$ & $\underline{\underline{z}} \overline{\underline{z}}$ & $\frac{1}{2}$ & $\frac{\vec{z}}{2}$ & 言言 & 坣 \\
\hline & $\underline{g}$ & & & & : & $\because \exists$ & 77 & & $\frac{n}{2}$ & $\nexists 2$ & 2 & & $\nexists \nexists$ & $\Rightarrow 7$ & & 䒠 & & $\overrightarrow{\underline{z}}$ & $\underline{\underline{\underline{z}}} \overline{\bar{z}}$ & $\frac{\vec{z}}{2}$ & $\mid \vec{z}$ & $\frac{\vec{z}}{\bar{z}} \overline{\bar{z}}$ & 竞 \\
\hline 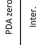 & $\frac{1}{2}$ & & & & 7 & 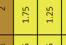 & 77 & & $\stackrel{n}{2}$ & 27 & 27 & & $\stackrel{7}{\cong}$ & 77 & & $\frac{\underline{\underline{z}}}{2}$ & $\frac{\vec{z}}{\underline{z}}$ & $\frac{\vec{z}}{\bar{z}}$ & $\underline{\underline{\underline{z}}} \overline{\underline{z}}$ & $\frac{1}{\frac{1}{2}} \frac{1}{2}$ & $\frac{\vec{z}}{2}$ & & $\frac{\vec{z}}{2}$ \\
\hline & \begin{tabular}{|l|l|} 
\\
\end{tabular} & $\circ$ & & 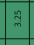 & $\%$ & $\therefore 7$ & 77 & & $\mathrm{~A}$ & 27 & 27 & & $\cong \approx$ & 77 & & 列 & & $\overline{\underline{z}}$ & $\frac{\bar{z}}{\bar{z}}$ & $\frac{\vec{z}}{2}$ & $\frac{\vec{z}}{\vec{z}}$ & & 考 \\
\hline & $\xi$ & 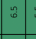 & & & $\frac{p}{7}$ & $-\because$ & 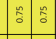 & & $\cong$ & $m$ & $\approx$ & & $\nexists \nexists$ & 77 & & 言 & 䇋 & $\overrightarrow{\underline{\underline{z}}}$ & $\overrightarrow{\underline{\underline{z}}}$ & 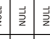 & $\vec{z}$ & 言言 & 志 \\
\hline $\mid \overline{\bar{z}}$ & 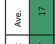 & $\Rightarrow:$ & & & \begin{tabular}{|l|l} 
\\
\\
\end{tabular} & $7:$ & 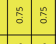 & & $\because 2$ & $m$ & $\approx$ & $\begin{array}{lll}n & n \\
\end{array}$ & $\stackrel{\because}{\because}$ & 77 & & $\frac{1}{2}$ & & $\frac{\vec{z}}{\bar{z}}$ & $\overrightarrow{\underline{z}} \overline{\underline{z}}$ & $\frac{1}{2} \frac{1}{2}$ & $\frac{\vec{t}}{2}$ & & $\frac{\vec{z}}{2}$ \\
\hline & \begin{tabular}{|l|}
5 \\
5
\end{tabular} & & & & 哭 & $\Rightarrow:$ & $5 \%$ & & $\ln 1$ & $m$ & $\approx$ & & $\cong \nexists$ & $7 \%$ & & 站 & 言 & $\frac{1}{\bar{z}}$ & $\frac{\bar{z}}{\bar{z}} \overline{\bar{z}}$ & & $\overrightarrow{\vec{z}}$ & & 坣 \\
\hline & & & & & $\frac{3}{\frac{3}{2}}=\frac{3}{2}=\frac{1}{2}$ & & & & 咅高 & 要费 & & 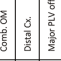 & & & & & & & & $\frac{\sqrt[c]{2}}{2}$ & & & \\
\hline & & & & & $\frac{Y}{3}$ & : & $\frac{g}{g}$ & & 表尊 & 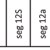 & & $\frac{8}{*}$ & 善善绕 & & & & & & & 善 & & & \\
\hline & & & & & & & & & & & & & & & & & & & & & & & \\
\hline
\end{tabular}

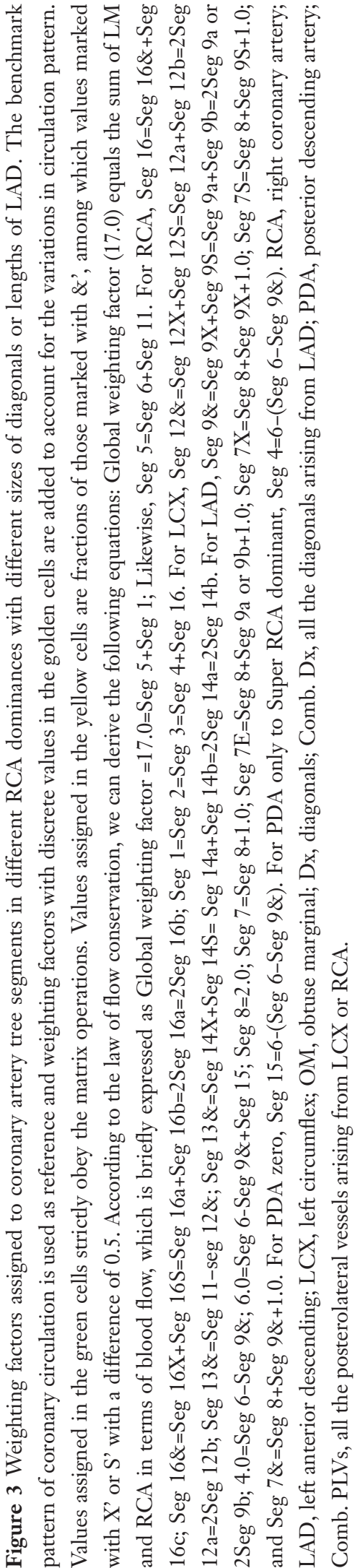




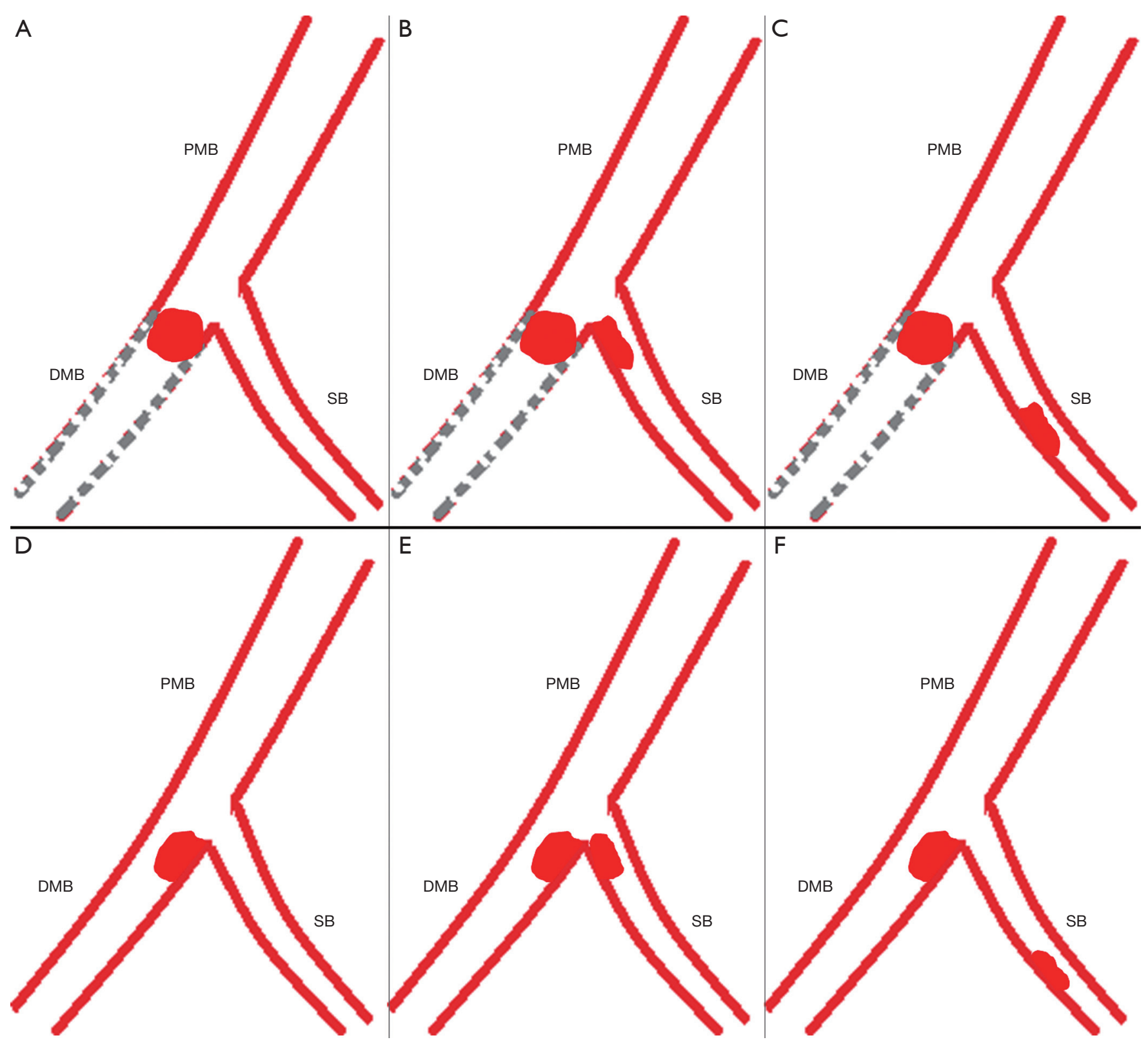

Figure 4 Lesion score modification within one segment in the presence of a significant SB in the 6 scenarios. The upper row is for the occluded lesion and the lower row is for the non-occluded lesion. PMB or DMB, proximal or distal major branches; SB, side branches. Dashed gray lines indicate occluded blood vessels distal to the occluded lesion.

the occlusive distal MB lesion. The scoring values for this segment should be modified for $7.0 \times 5-3.0 \times 5=20.0$ using formula: $\mathrm{S}=$ subtotal score $-\mathrm{W}$ SBs $\times 5$. All the vessels are required to be modified if appropriate regardless of their sizes. Six scenarios required to be modified according to the corresponding formulas were elaborated online (http: // www.catletscore.com).

\section{Software-based CatLet score algorithm}

The CatLet score calculation is feasible by use of a computer program consisting of sequential and interactive self-guided questions. The calculator and its elaborate tutorial are available at http://www.catletscore.com. The IE browser is required to run this calculator. 
Table 1 Scoring or describing of lesion's adverse characteristics

\begin{tabular}{|c|c|}
\hline Characteristics & Values \\
\hline RCA dominance & 6 types \\
\hline Dx size & 3 types \\
\hline LAD length & 3 types \\
\hline \multicolumn{2}{|l|}{ Diameter reduction } \\
\hline Total occlusion & $\times 5$ \\
\hline Significant lesion (50-99\% diameter reduction) & $\times 2$ \\
\hline \multicolumn{2}{|l|}{ Lesion types } \\
\hline Native non-occlusion & Yes or No \\
\hline In-stent non-occlusion & Yes or No \\
\hline Native ATO & Yes or No \\
\hline In-stent ATO & Yes or No \\
\hline Native CTO & Yes or No \\
\hline In-stent CTO & Yes or No \\
\hline Stented vessels & Yes or No \\
\hline Ballooned vessels & Yes or No \\
\hline Calcified vessels & Yes or No \\
\hline “Culprit” lesion judgment & Yes or No \\
\hline Trifurcations & Yes or No \\
\hline Bifurcations & Yes or No \\
\hline Medina types & Yes or No \\
\hline Angulation $<70$ & Yes or No \\
\hline Aorto ostial stenosis & Yes or No \\
\hline Severe tortuosity & Yes or No \\
\hline Length $>20 \mathrm{~mm}$ & Yes or No \\
\hline Heavy calcification & Yes or No \\
\hline Thrombus & Yes or No \\
\hline Anatomical description of native coronary system & Yes or No \\
\hline
\end{tabular}

Adverse characteristics are defined as in the SYNTAX score. However, we only record these characteristics as "Yes" or "No", and do not assign values to them, which are different from the SYNTAX score (9). In addition, we replace the "Diffuse disease/ small vessels" with anatomical description of native coronary trees. Stented, ballooned or calcified vessels indicate those ever treated or "calcified" vessels with non-significant lesions now ( $<50 \%$ stenosis). Trifurcation or bifurcation is according to the actual states of blood vessels involved, but not limited to the segment junctions designated in the SYNTAX score (9). RCA, right coronary artery; $L A D$, left anterior descending artery; ATO, acute total occlusion; CTO, chronic total occlusion.

The algorithm includes 15 main questions as listed in Table 1. The first question includes 3 sub-questions: RCA dominance, Dx size and LAD length. Answering to these 3 sub-questions will define one of 54 types of coronary artery circulation proposed in this angiographic scoring system. The second question includes two options: option 1 is for recording normal coronary artery anatomy; and option 2 (most important) is for evaluating the lesions. Question 3 addresses the lesion-based unlimited segment selection. Question 4 addresses 9 lesion types: (I) native nonocclusion; (II) in-stent non-occlusion; (III) native acute total occlusion (ATO); (IV) in-stent ATO; (V) native chronic total occlusion (CTO); (VI) in-stent CTO; (VII) stented vessels; (VIII) ballooned vessels; and (IX) calcified vessels. We will elaborate on the scoring algorithm immediately below with non-occlusion or ATO lesions as examples. For other lesion types, you can visit the online tutorial.

For non-occlusion or ATO lesions, questions 5-11 address the lesion-based evaluation, where we qualitatively record the adverse characteristics as "Yes" or "No" in our scoring system. Question 12-14 addresses treatments, among which the stenting strategy is classified according to the MADS classification recommended by European Bifurcation Club (13). Question 15 addresses scoring modification if necessary. Upon completion of question 15 , the algorithm proceeds to the last question or to another cycle of "add another lesion". The last question of the CatLet score algorithm is to describe the anatomy of the native coronary trees. For a patient with coronary artery disease, it takes around 5-10 minutes to process a patient depending on the complexity and severity of coronary artery disease. Figure 5 showed scoring of a lesion on proximal LAD according to the CatLet score algorithm.

\section{Validation}

The utility of this CatLet score for predicting outcomes and standardizing the angiographic data collection will be investigated in a series of clinical trials enrolling "all-comers" with CAD.

It will be evaluated for outcome predictions in a historic or prospective cohort of "all-comers" with acute myocardial infarction, unstable angina pectoris, and CTO in a single center or multi-center studies.

The first validation study has been conducted in acute myocardial infarction and registered at http://www. chictr.org.cn (ChiCTR-POC-17013536), which will be published soon.

\section{Summary}

The most important characteristics of this CatLet 


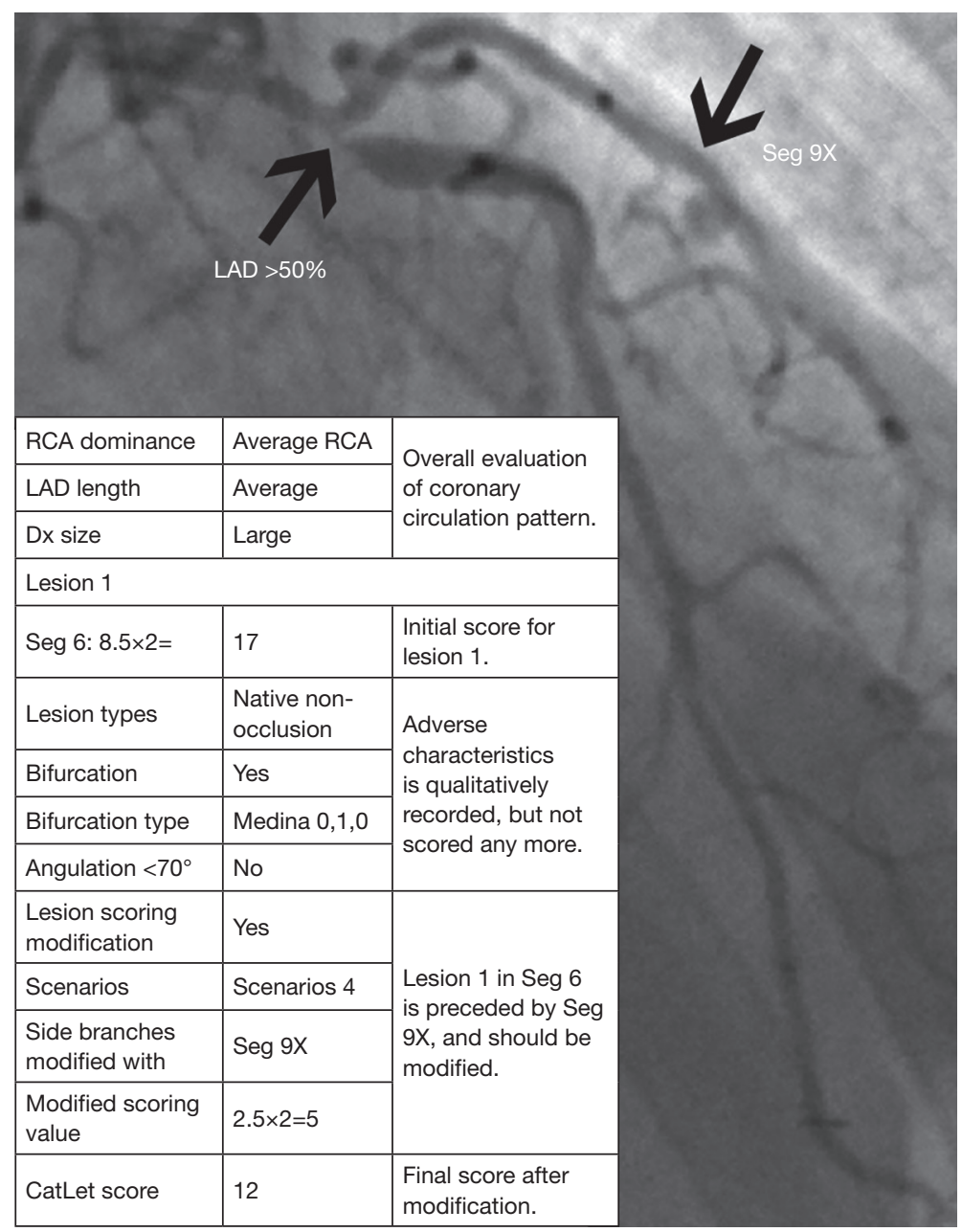

Figure 5 Scoring of a sample patient on proximal LAD according to the CatLet score algorithm. CatLet, Coronary Artery Tree description and Lesion EvaluaTion; LAD, left anterior descending artery.

angiographic scoring system to be emphasized is that it is focusing on the variability in the coronary anatomy.

The ultimate goal is to create a new coronary angiographic scoring tool so as to (I) accommodate the variability in the coronary anatomy; (II) to grade the severity and complexity of the coronary artery disease; (III) to standardize the coronary angiographic data collection and to facilitate the comparison and exchange of these data between different catheter labs; and, finally; (IV) to predict clinical outcomes in patients with CAD.

\section{Acknowledgments}

Funding: This work was in part supported by the Sci-Tech
Development Program of Suzhou City (SYS2019040 to He YM).

\section{Footnote}

Conflicts of Interest: The authors have no conflicts of interest to declare.

Ethical Statement: The authors are accountable for all aspects of the work in ensuring that questions related to the accuracy or integrity of any part of the work are appropriately investigated and resolved. This study complies with the Declaration of Helsinki and has full local approval for using subject data. 


\section{References}

1. De Bruyne B, Pijls NH, Kalesan B, et al. Fractional flow reserve-guided PCI versus medical therapy in stable coronary disease. N Engl J Med 2012;367:991-1001.

2. van Nunen LX, Zimmermann FM, Tonino PA, et al. Fractional flow reserve versus angiography for guidance of PCI in patients with multivessel coronary artery disease (FAME): 5-year follow-up of a randomised controlled trial. Lancet 2015;386:1853-60.

3. Pijls NH, Fearon WF, Tonino PA, et al. Fractional flow reserve versus angiography for guiding percutaneous coronary intervention in patients with multivessel coronary artery disease: 2-year follow-up of the FAME (Fractional Flow Reserve Versus Angiography for Multivessel Evaluation) study. J Am Coll Cardiol 2010;56:177-84.

4. Leaman DM, Brower RW, Meester GT, et al. Coronary artery atherosclerosis: severity of the disease, severity of angina pectoris and compromised left ventricular function. Circulation 1981;63:285-99.

5. Goel PK, Khanna R, Batra A, et al. Syntax score: The fallacies and remedies-A perspective. World J Cardiovasc Dis 2013;3:448-53.

6. Serruys PW, Unger F, van Hout BA, et al. The ARTS study (Arterial Revascularization Therapies Study). Semin Interv Cardiol 1999;4:209-19.

7. Cerqueira MD, Weissman NJ, Dilsizian V, et al. Standardized myocardial segmentation and nomenclature for tomographic imaging of the heart. A statement for healthcare professionals from the Cardiac Imaging Committee of the Council on Clinical Cardiology of the American Heart Association. Circulation 2002;105:539-42.

8. Ormiston JA, Kassab G, Finet G, et al. Bench testing and coronary artery bifurcations: a consensus document from the European Bifurcation Club. EuroIntervention 2018;13:e1794-803.

9. Sianos G, Morel MA, Kappetein AP, et al. The SYNTAX Score: an angiographic tool grading the complexity of coronary artery disease. EuroIntervention 2005;1:219-27.

10. Austen WG, Edwards JE, Frye RL, et al. A reporting system on patients evaluated for coronary artery disease. Report of the Ad Hoc Committee for Grading of Coronary Artery Disease, Council on Cardiovascular Surgery, American Heart Association. Circulation 1975;51:5-40.

11. Holman BL, Adams DF, Jewitt D, et al. Measuring regional myocardial blood flow with $133 \mathrm{Xe}$ and the Anger camera. Radiology 1974;112:99-107.

12. Gallik DM, Obermueller SD, Swarna US, et al. Simultaneous assessment of myocardial perfusion and left ventricular function during transient coronary occlusion. J Am Coll Cardiol 1995;25:1529-38.

13. Lassen JF, Holm NR, Banning A, et al. Percutaneous coronary intervention for coronary bifurcation disease: 11th consensus document from the European Bifurcation Club. EuroIntervention 2016;12:38-46.
Cite this article as: $\mathrm{Xu} M X$, Teng RL, Ruddy TD, Schoenhagen P, Bartel T, Di Bartolomeo R, Aksoy O, Desai M, von Kodolitsch Y, Escaned J, McCullough PA, Vasudevan A, Shen CX, Zhao X, Zhou YF, Xu HF, Cheng XJ, He YM; written on behalf of the AME Interventional Cardiology Collaborative Group. The CatLet score: a new coronary angiographic scoring tool accommodating the variable coronary anatomy for the first time. J Thorac Dis 2019;11(12):51995209. doi: 10.21037/jtd.2019.12.18 